\title{
Control de presiones venosa y arterial para evitar la disfunción del acceso vascular y su influencia en la dosis de diálisis
}

\author{
Mª Luz Sánchez Tocino, Silvia Villoria González, Belén Muñoz García, Alberto Sánchez Martín \\ Fundación Renal Íñigo Álvarez de Toledo (FRIAT). Centros Hemodiálisis "Las Encinas" Ciudad Rodrigo y "El Castañar" \\ Béjar. Salamanca. España
}

\section{Resumen}

Introducción: Las guías clínicas del acceso vascular, recomiendan el seguimiento y monitorización de la fistula arteriovenosa y recientes estudios el mantenimiento de la presión venosa y presión arterial dentro de unos límites seguros.

Objetivo: Analizar las pautas de monitorización de diálisis en nuestros pacientes y ajustarlas para conseguir presiones dinámicas seguras.

Valorar como afecta a la dosis de diálisis y realizar las modificaciones necesarias para recuperar esta de forma individualizada.

Material y métodos: Se recogen datos de 29 pacientes, en tres periodos de tiempo de 15 sesiones; Periodo 0: pauta de diálisis habitual; Periodo 1: Flujo de bomba protector no modificable; Periodo 2: Flujo de bomba según presiones diarias y se modifica la pauta de diálisis. Se analizan las variables; flujo de bomba, presiones venosa, arterial y dosis de diálisis medido a través del Kt.

Resultados: En el periodo 0, el $83 \%$ de los pacientes presenta una presión venosa $>160 \mathrm{~mm} \mathrm{Hg}$ y $21 \%$ presión arterial $<-200 \mathrm{~mm} \mathrm{Hg}$. En el periodo 1, tras la intervención sobre el flujo, $20 \%$ de pacientes tiene presión venosa > de $160 \mathrm{~mm} \mathrm{Hg}$ y 3,4\% arterial < $-200 \mathrm{~mm} \mathrm{Hg}$. En el periodo 3 el 100\% pacientes están con presiones seguras. En relación a Kt: periodo

Correspondencia:
$M^{\mathrm{a}}$ Luz Sánchez Tocino
Centro de Hemodiálisis "Las Encinas"
C/ Escuelas, $\mathrm{n}^{\circ}$ 4-6. 37500 Ciudad Rodrigo
Salamanca. España
E-mail: Isanchez@friat.es

0 media de 49I, periodo 1 media 46,81 y se recupera periodo 2 a 50,65I $(p<0,001)$. Para ello en el periodo 2 se realizaron 21 intervenciones a 18 pacientes de forma individualizada.

Conclusión: Se puede dializar siguiendo las últimas recomendaciones de límites de presiones dinámicas manteniendo la dosis de diálisis y actuando sobre otros parámetros de la pauta de manera individualizada.

PALABRAS CLAVE

- PROTECCIÓN DEL ACCESO VASCULAR

- FLUJO DE BOMBA

- PRESIÓN VENOSA

- PRESIÓN ARTERIAL

- DOSIS DIÁLISIS

Venous and arterial pressure control to avoid vascular access dysfunction and his influence in the dyalisis dose

\begin{abstract}
Introduction: Clinical guidelines vascular access, recommend tracking and monitoring arteriovenous fistula and recent studies maintaining maintaining venous and arterial pressure in safe limits.

Objective: To analyse dialysis monitoring patterns in our patients and to adjust them in order to get safe dynamic pressures.

To value the influence in the dialysis dose and to carry out the required changes to restore it in an individualized way.
\end{abstract}


Materials and methods: Dates of three periods in 29 patients has been taken in. Period zero: Usual dialysis pattern: Period one: Protective blood flow not changeable. Period two: Blood flow according to diary pressure and modified dialysis pattern. The variables analyzed are; blood flow, venous and arterial pressure, dialysis dose according to the Kt.

Results: In period zero, $83 \%$ of the patients present a venous pressure $>160 \mathrm{~mm} \mathrm{Hg}$ and the $21 \%$ an arterial pressure $<-200 \mathrm{~mm} \mathrm{Hg}$. In period one after the action above the flow $20 \%$ of the patients has a venous pressure $>$ de $160 \mathrm{~mm} \mathrm{Hg}$ and $3.4 \%$ an arterial pressure $<-200 \mathrm{~mm} \mathrm{Hg}$. In period three, the $100 \%$ of the patients have safe pressures. In relation with the $\mathrm{Kt}$, it was mean of 491 in the period zero, 46,81 in the period one and it regain in the period two to $50,651(p<0,001)$. For that in the period two, 21 individualized interventions in 18 patients were performed.

Conclusions: It is possible to dialyze according to the last guidelines in dynamic pressure limits, keeping the dialysis dose and acting in others parameters of the individualized pattern.

\section{KEYWORDS}

- VASCULAR ACCESS PROTECTED

- BLOOD FLOW

- VENOUS PRESSURE

- ARTERIAL PRESSURE

- DIALYSIS DOSE

\section{Introducción}

El acceso vascular (AV) constituye uno de los elementos imprescindibles para poder llevar a cabo el tratamiento con hemodiálisis. El acceso vascular ideal debe reunir al menos tres características: Permitir el abordaje seguro y continuado al sistema vascular, proporcionar flujos suficientes que permitan suministrar la dosis de diálisis programada y estar exento de complicaciones ${ }^{1}$.

En la actualidad, la fístula arteriovenosa (FAV), es el acceso vascular que más se acerca a estos requisitos y por lo tanto constituye la primera vía de elección para el inicio del tratamiento con hemodiálisis, dada su elevada supervivencia ${ }^{2,3,4}$.
En este sentido, las diferentes guías clínicas del AV recomiendan la aplicación periódica de programas activos de seguimiento y monitorización del mismo ${ }^{4,5}$.

Junto con la exploración física, el control de las presiones dinámicas, presión arterial prebomba (PA) y presión venosa del circuito (PV), durante la sesión es el primer eslabón en el seguimiento del $\mathrm{AV}$, siendo el enfermero el encargado de este procedimiento $0^{6,7}$. Son varios los trabajos que afirman que a mayor PV y menor PA disminuye la supervivencia del acceso $8,9,10,11,12$; sin embargo no hay una cifra límite de presiones que se establezca como segura, si bien parece que se recomienda no sobrepasar los $-190 \mathrm{mmHg}$ para la $\mathrm{PA}^{11}$ y en cuanto a la PV, según recientes estudios ${ }^{12}$, se recomienda bajar los límites aceptados como normales de $200 \mathrm{mmHg}$ a $150 \mathrm{mmHg}$ para aumentar la supervivencia del AV a largo plazo. Así el mantenimiento del control de presiones dinámicas como factor de riego del $\mathrm{AV}$, nos obliga a controlar el flujo de bomba (Qb). Este, junto con el tiempo de la sesión, el aclaramiento de urea del dializador y el flujo del líquido dializante, son algunos de los factores implicados en la dosis de diálisis ${ }^{13}$, de manera que al modificar nuestros flujos, la eficacia dialítica podría verse afectada y requerir nuevos ajustes en la pauta de diálisis.

El objetivo del presente estudio fue evaluar las presiones dinámicas de nuestros pacientes, adaptarlas a las últimas recomendaciones sobre el límite de las mismas y analizar cómo afectó el control de presiones sobre el Qb y a su vez sobre la dosis de diálisis.

\section{Material y métodos}

Se realizó un estudio prospectivo sobre todos los pacientes con enfermedad renal crónica en programa de hemodiálisis convencional y portadores de fistula arteriovenosa nativa o protésica una vez superado el periodo de maduración, en los dos centros periféricos que la Fundación Renal Íñigo Álvarez de Toledo tiene en la provincia de Salamanca.

\section{Variables de estudio:}

Variables primarias: Flujo de bomba (Qb), Presión venosa (PV) y presión arterial(PA) y KT medido mediante dialisancia iónica OCM Fresenius.

Variables secundarias: Variables demográficas (edad, sexo y etiología de la ERC). Variables relacionadas con la diálisis (Tiempo en diálisis, tipo acceso vascular y tiempo del mismo, dializador, flujo de baño (Qd) y calibre de agujas). 
Según las últimas recomendaciones ${ }^{11,12}$, se consideró como límites seguros para el $\mathrm{AV}$, dializar con $\mathrm{PV}$ máximas de $160 \mathrm{~mm} \mathrm{Hg}$ y PA mínimas de $-200 \mathrm{~mm} \mathrm{Hg}$, redondeando la cifra de $150 \mathrm{~mm} \mathrm{Hg}$ y $190 \mathrm{~mm} \mathrm{Hg}$ referente en la bibliografía, ya que se utilizaron monitores FRESENIUS 4008 y el display de lectura marcará valores 140 ó $160 \mathrm{~mm} \mathrm{Hg}$ en caso de PV y -180 ó $-200 \mathrm{~mm} \mathrm{Hg}$ en caso de PA.

Para valorar la dosis de diálisis se utilizó la medida del Kt. Lowrie y col recomiendan un Kt mínimo de 40-45 litros en las mujeres y de 45-50 litros en los hombres ${ }^{14}$, posteriormente esto quedará validado, considerándose el Kt un buen marcador de la dosis de diálisis ${ }^{15}$, incluso algunos autores lo señalan como el mejor indicador en situación de infradiálisis ${ }^{16}$, además desde un punto de vista de la actuación enfermera, la medición continua de la dosis mediante el Kt permite conocer la eficacia en cada sesión sin necesidad de realizar extracciones sanguíneas seriadas ${ }^{17}$.

Para el análisis de los resultados de KT se dividió por tanto la muestra en función del sexo, y se establecieron 4 grupos de valores:

- Grupo 1 pacientes con kt menor de 401.

- Grupo 2 pacientes con kt entre 40-45I.

- Grupo 3 pacientes con kt entre 45-50I.

- Grupo 4 pacientes con kt mayor de 501.

Se determinaron tres periodos de tiempo a analizar:

Periodo 0: 15 sesiones por paciente sin modificar parámetros de flujo del $A V$, en las que el $Q b$ estuvo a criterio de enfermería, midiéndose $Q b, P V$ y PA y kt obtenido.

Periodo 1: 15 sesiones por paciente, en el que se pautó un flujo de bomba no modificable, que se estableció valorando durante tres sesiones que las presiones venosas y arteriales estuvieran dentro de los límites definidos anteriormente como seguros. En este periodo se mantuvo el resto de pauta de $\mathrm{Hd}$ del periodo 0: Membrana, tiempo de $\mathrm{Hd}$, flujo de baño, calibre de las agujas.

Periodo 2: 15 sesiones en las que se ajustó el Qb en las distintas sesiones para no sobrepasar en ningún caso PV $160 \mathrm{~mm} \mathrm{Hg}$ o PA $-200 \mathrm{~mm} \mathrm{Hg}$ y además se modificó la pauta de diálisis (tiempo de diálisis, calibre de las agujas, tamaño de la membrana, flujo de baño), en función a los cambios en la dosis de diálisis (medidos por $\mathrm{Kt}$ ) producidos entre el periodo 0 y 1 , de manera individual y según las características personales de cada paciente.
Los parámetros de estudio se recogieron en la historia clínica informática mediante el programa Nefrosoft ${ }^{\circledR}$ V.6.5.3.

EI análisis estadístico se ejecutó con el programa SPSS 15.0 para Window 10 . Se realizó un estudio descriptivo de los principales datos demográficos, se utilizó como medidas de dispersión central en algunos casos la media y desviación estándar o la mediana y el rango según el comportamiento normal de las variables. Se comparó las distintas variables del estudio como datos pareados de cada paciente en los distintos periodos del estudio. Se concretó el análisis de significación estadística para $\mathrm{p} \leq 0,05$ utilizando pruebas no paramétricas con test de Friedman para diferencias intraperiodos y test de rangos de signos de Wilcoson para diferencias interperiodos ya que muchas de las variables no siguieron una distribución normal.

\section{Resultados}

Se incluyeron 29 pacientes, los datos demográficos principales se describen en tabla 1.

Tabla 1. Características pacientes en diálisis y acceso vascular.

\begin{tabular}{lc}
\hline Parámetro & Valor \\
\hline Edad (mediana), años & $\mathbf{7 2}$ (rango 24-92) \\
\hline Sexo, $\mathbf{n}(\%)$ & $19(65,5)$ \\
Hombre & $10(34,5)$ \\
\hline Mujer & $43,9($ rango $7-118)$ \\
\hline Permanencia en HD, meses & \\
\hline Causa de insuficiencia renal, $\mathbf{n}(\%)$ & $9(31)$ \\
\hline Diabetes & $5(17)$ \\
\hline Glomerular & $3(10)$ \\
\hline Vascular & $4(14)$ \\
\hline Intersticial & $5(17)$ \\
\hline Desconocida & $3(10)$ \\
\hline Otras & \\
\hline Tipo de acceso vascular, $\mathbf{n}(\%)$ & $26(89,7)$ \\
\hline FAV nativa & $3(10,3)$ \\
\hline FAV protésica & \\
\hline Permanencia del AV, meses & $38,1 \pm 30,7($ rango $7-123)$ \\
\hline
\end{tabular}

El análisis descriptivo de las variables en estudio en los distintos periodos, se detallaron en la tabla 2. En esta misma tabla se analizó la variación del Kt en los distintos periodos. 
Tabla 2. Análisis descriptivo de las variables a estudio.

\begin{tabular}{|c|c|c|c|c|c|c|c|}
\hline & & \multicolumn{2}{|l|}{ Periodo 0} & \multicolumn{2}{|l|}{ Periodol } & \multicolumn{2}{|l|}{ Periodo 2} \\
\hline Flujo & $\begin{array}{l}\text { Mediana } \\
\text { Rango }\end{array}$ & \multicolumn{2}{|c|}{$\begin{array}{l}389,7 \\
323,6-426,6\end{array}$} & \multicolumn{2}{|l|}{$\begin{array}{l}358,7 \\
280-400\end{array}$} & \multicolumn{2}{|c|}{$\begin{array}{l}381,3 \\
288,6-482\end{array}$} \\
\hline PV & $\begin{array}{l}\text { Mediana } \\
\text { Rango } \\
\mathrm{N}, \% ; \mathrm{PV}>160 \mathrm{~mm} \mathrm{Hg}\end{array}$ & \multicolumn{2}{|c|}{$\begin{array}{l}174 \\
135,8-198,8 \\
24(82,8)\end{array}$} & \multicolumn{2}{|c|}{$\begin{array}{l}159 \\
129,8-170,9 \\
6(20,4)\end{array}$} & \multicolumn{2}{|c|}{$\begin{array}{l}159,5 \\
124,3-161,3 \\
1(3,4)\end{array}$} \\
\hline PA & $\begin{array}{l}\text { Mediana } \\
\text { Rango valor es negativo } \\
\mathrm{N}, \% \text {; PA }<-200 \mathrm{~mm} \mathrm{Hg}\end{array}$ & \multicolumn{2}{|c|}{$\begin{array}{l}-187,3 \\
{[148,5-210,5]} \\
6(20,7)\end{array}$} & \multicolumn{2}{|c|}{$\begin{array}{l}-173,7 \\
{[108,9-206,8]} \\
1(3,4)\end{array}$} & \multicolumn{2}{|c|}{$\begin{array}{l}-177,8 \\
{[111,7-194,3]} \\
0\end{array}$} \\
\hline \multirow[t]{6}{*}{$\mathrm{Kt}$} & $\begin{array}{l}\text { Mediana } \\
\text { Rango }\end{array}$ & \multicolumn{2}{|l|}{$\begin{array}{l}49,3 \\
37-63,9\end{array}$} & \multicolumn{2}{|c|}{$\begin{array}{l}46,8 \\
38,7-57,8\end{array}$} & \multicolumn{2}{|c|}{$\begin{array}{l}49,6 \\
40,5-65,4\end{array}$} \\
\hline & Grupos Kt n,\% & Hombres & Mujeres & Hombres & Mujeres & Hombres & Mujeres \\
\hline & $<40$ & 0 & $1(10)$ & 0 & $2(20)$ & 0 & 0 \\
\hline & $40-45$ & $2(10,5)$ & $5(50)$ & $4(21,1)$ & $4(40)$ & 0 & $2(20)$ \\
\hline & $45-50$ & $8(42,1)$ & 0 & $8(42,1)$ & $2(20)$ & $9(47,4)$ & $4(40)$ \\
\hline & $>50$ & $9(47,4)$ & $4(40)$ & $7(36,8)$ & $2(20)$ & $10(52,6)$ & $4(40)$ \\
\hline
\end{tabular}

Fuente: Datos de la investigación, 2015.

En el periodo 0, el $82,7 \%$ de los pacientes se dializaron con cifras de PV por encima de $160 \mathrm{~mm} \mathrm{Hg}$ y el $20,7 \%$ con cifras de PA superiores a $-200 \mathrm{~mm} \mathrm{Hg}$.

Al bajar los flujos para controlar las presiones dinámicas, la eficacia dialítica descendió de 49,3 I a 46.8 I en el periodo 1 y se recuperó hasta 49.6 I en el periodo 2 y estas diferencias fueron estadísticamente significativas $(p<0,001)$, entre los 3 periodos. Las diferencias fueron especialmente importantes entre el periodo 0 y periodo 1 y entre el periodo 1 y $2(p<0,001)$, rozando la significación entre el periodo 0 y $2 p=0,05$.

En el periodo 2, tras la intervención sobre otros factores de la pauta de diálisis, el $100 \%$ de los varones se han dializado por encima de $45 \mathrm{I}$, el $80 \%$ de las mujeres por encima del $45 \mathrm{I}$ y el $100 \%$ de las mujeres por encima de $40 \mathrm{I}$.

Se estudiaron, las diferencias significativas en los cambios de PV y PA realizados tras el ajuste de flujos y como estas se han mantenido en el periodo 2 tras la intervención (tabla 3).

También existieron diferencias en el Qb entre el periodo 0 y el periodo 1 en el que bajan los flujos y como estos se recuperaron con la intervención realizada, siendo las diferencias entre el periodo basal y el final no significativas.
En cuanto las intervenciones sobre la pauta habitual realizadas entre el periodo 1 y 2 , se realizaron 21 intervenciones sobre los 29 pacientes cuya distribución se refleja en el Gráfico 1.

Tabla 3. Significación estadística prueba de los rangos con signos de Wilconson para datos pareados de las variables en estudio intraperiodos.

\begin{tabular}{|c|c|c|c|c|}
\hline & $\begin{array}{l}\text { Significación } \\
\text { estadística }\end{array}$ & Periodo 0-1 & Periodo 1-2 & Periodo 0-2 \\
\hline $\mathbf{Q b}$ & & $p<0,001$ & $p=0,004$ & $p=0,2$ \\
\hline PV & & $p<0,001$ & $p=0,642$ & $p<0,001$ \\
\hline PA & & $p<0,001$ & $p=0,567$ & $p<0,001$ \\
\hline Kt & & $p<0,001$ & $p<0,001$ & $p=0,05$ \\
\hline
\end{tabular}

Flujo de bomba (Qb), Presión venosa en $\mathrm{mm} \mathrm{Hg}(\mathrm{PV})$, Presión arterial en $\mathrm{mm} \mathrm{Hg}(\mathrm{PA})$, Dosis de diálisis en I (Kt).

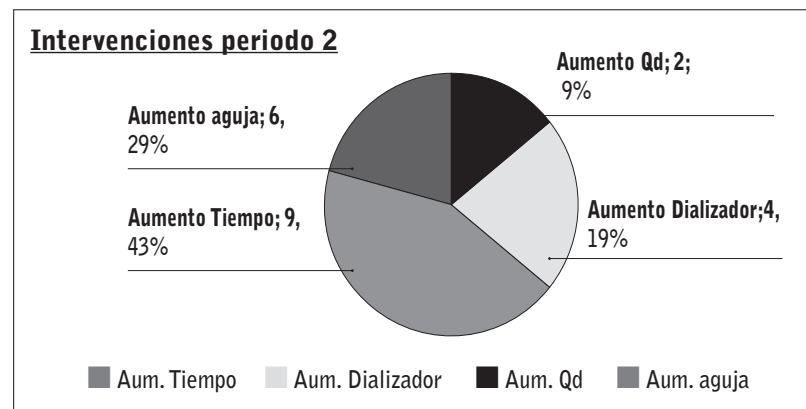

Gráfico 1. Tipo de intervenciones realizadas sobre la pauta habitual entre el periodo 1 y periodo 2 . 
Tabla 4. Intervenciones realizadas sobre la pauta habitual entre el periodo 1 y periodo 2 .

\begin{tabular}{|c|c|c|}
\hline & $\begin{array}{l}\text { Periodo } 0 \\
\text { y Periodo } 1\end{array}$ & Periodo 2 \\
\hline $\begin{array}{l}\text { Tiempo de sesión HD, } \\
\text { m/horas semanales }\end{array}$ & $667,24 \mathrm{~m} / 11,12 \mathrm{~h}$ & $685,86 \mathrm{~m} / 11,43 \mathrm{~h}$ \\
\hline \multicolumn{3}{|l|}{ Dializador, n (\%) } \\
\hline Helixona $1.8 \mathrm{~m}^{2}$ & $12(41,38)$ & $12(41,38)$ \\
\hline Polinefrona $1.9 \mathrm{~m}^{2}$ & $14(48,27)$ & $10(35,47)$ \\
\hline Polinefrona $2.1 \mathrm{~m}^{2}$ & $2(6,90)$ & $6(20,69)$ \\
\hline Polinefrona $2.5 \mathrm{~m}^{2}$ & $1(3,45)$ & $1(3,45)$ \\
\hline \multicolumn{3}{|l|}{ Flujo de baño, n (\%) } \\
\hline $500 \mathrm{ml} / \mathrm{m}$ & $29(100)$ & $27(93,1)$ \\
\hline $800 \mathrm{ml} / \mathrm{m}$ & 0 & $2(6,9)$ \\
\hline \multicolumn{3}{|l|}{ Tipo de aguja, n(\%) } \\
\hline $16 G$ & $5(17.3)$ & $3(10,35)$ \\
\hline 15G & $24(82,7)$ & $22(75,86)$ \\
\hline $14 G$ & 0 & $4(13,79)$ \\
\hline
\end{tabular}

Las pautas de los pacientes en los tres periodos quedaron descritas en la tabla 4.

\section{Discusión}

El control de fístula arteriovenosa (FAV) es fundamental para los pacientes en $\mathrm{HD}^{4,5}$. Dentro de la monitorización del $\mathrm{AV}$, enfermería debe controlar las presiones dinámicas ( $P V$ y $P A$ ) durante el tratamiento con el fin de proteger la supervivencia del AV. Una parte importante de la evaluación de estos parámetros reside en la evolución de los mismos a lo largo del tiempo, dado que varían según las características del paciente y del $A \mathrm{~V}^{4,7}$. Teniendo esto en cuenta, son varios los trabajos que afirman que a mayor PV y me-

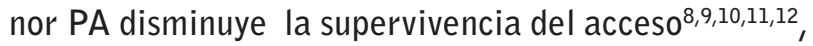
pero no hay una cifra límite establecida como segura. En este sentido algunos autores relacionan una PA negativa menor de $-190 \mathrm{~mm} \mathrm{Hg}$ con un descenso de la supervivencia del acceso vascular ${ }^{11}$. Esto se explica porque la hiperplasia intimal, lesión precursora de la estenosis y trombosis del acceso vascular, se origina por las vibraciones y turbulencias relacionadas con el $\mathrm{Qb}^{18}$, y la PA pre bomba anormalmente elevada puede contribuir a la lesión endotelial por la excesiva succión y hemólisis ${ }^{19}$. En cuanto a la PV, en la bibliografía tampoco existen unos límites claros, si bien según un reciente estudio ${ }^{12}$, se recomienda bajar los límites aceptados como normales de $200 \mathrm{~mm} \mathrm{Hg}$ a $150 \mathrm{~mm}$ $\mathrm{Hg}$.

En este estudio se analizó el porcentaje de pacientes que se estaban dializando con valores de PV y PA fuera de los límites aceptados actualmente en la bibliografía. Tras el análisis se comprobó que en muchos de los pacientes se aceptaban cifras sobre todo de PV superiores a las recomendadas. A pesar de bajar los flujos de bomba, durante el periodo 1 no en todos los pacientes las presiones descendieron a límites seguros, 6 pacientes mantuvieron cifras de PV superiores a $160 \mathrm{~mm} \mathrm{Hg}$ y en un caso la PA se mantuvo por debajo de $-200 \mathrm{~mm}$ $\mathrm{Hg}$. Hay que tener en cuenta que los parámetros hemodinámicos pueden verse afectados entre otras cosas por cambios en la zona de punción, mal posición de las agujas, pinzamiento de los sistemas, viscosidad de la sangre e hipotensión arterial ${ }^{4,7}$, nuestras medianas se vieron afectadas por variaciones en sesiones puntuales, esto quedó corregido en el tercer periodo en el que no se establece una pauta fija de flujo, sino que dependerá de las circunstancias de la sesión, de manera que en el mismo, la totalidad de los pacientes se dializan en límites aceptados.

La dosis administrada de diálisis influye en la supervivencia del paciente en hemodiálisis ${ }^{20,21}$. Entre los factores implicados en alcanzar dicha dosis se encuentran el tiempo de la sesión, el flujo sanguíneo efectivo, el aclaramiento de urea del dializador y el flujo del líquido dializante ${ }^{13}$. En este estudio mayoritariamente disminuyeron los flujos de bomba y esto ha afectado a la dosis de diálisis de nuestros pacientes, medida por $\mathrm{Kt}$, de manera estadísticamente significativa.

Si bien queremos mantener en buen estado de nuestros accesos vasculares, sabemos de la importancia de conseguir una diálisis adecuada. En nuestro estudio tras la pérdida de eficacia dialítica al bajar los flujos se valoró de manera individual el cambio de pauta más adecuado según las circunstancias de cada paciente. Se realizaron 21 intervenciones sobre los 29 pacientes, la mayoría referente al tiempo de diálisis que aumenta en 9 pacientes, como sabemos, el tiempo de tratamiento, es el elemento más importante y siempre eficaz sobre el que podemos influir para mejorar la dosis. Las recomendaciones de las guías europeas y españolas son de un tiempo alrededor de 12 horas semanales ${ }^{22,23}$. En nuestro caso de paso de $11,1 \mathrm{~h}$ a $11,43 \mathrm{~h}$ semanales obteniéndose un aumento significativo del Kt en todos los casos. En cuanto al tamaño de la agujas se realizó en 6 pacientes, a pesar de la controversia en la bibliografía 
del uso de agujas $14 \mathrm{G}$ en cuanto al aumento del dolor y sangrado respecto al aumento de la eficacia ${ }^{24,25}$, nosotros aumentamos el tamaño, lo cual nos permitió aumentar los flujos de nuevo incluso a valores mas altos que el periodo 0 , manteniendo cifras de presiones seguras. Igualmente a pesar de que recientes publicaciones cuestionan el aumento del flujo de baño frente al aumento del tiempo en relación al ahorro del gasto 26,27 son varios los autores que refieren un aumento de la eficacia de diálisis de un $5-10 \%{ }^{28,29}$, en dos de nuestros pacientes se aumentó de 500 a 800 el Qb, ya que esta se valoró como la mejor opción de aumento de eficacia en estos dos casos. Por último se sabe que la superficie y permeabilidad de cada dializador se expresa por su coeficiente de trasferencia de masas (KoA), a mayor superficie, mayor trasferencia de masas. Actualmente se usan superficies de $1.5-2.5 \mathrm{~m}^{2}$, según la superficie corporal de paciente29, en nuestro estudio se ha aumentado el tamaño del dializador en 4 casos de $1.9 \mathrm{~m}^{2}$ a $2.1 \mathrm{~m}^{2}$.

Los cambios realizados en la pauta habitual referentes al tiempo de tratamiento, flujo de baño, membrana o tamaño de las agujas, no sólo hicieron que se recuperaran las dosis de Kt que había disminuido con los ajustes del flujo sino que además se alcanzaran cifras de dosis de diálisis recomendada para todos los pacientes.

Hay que tener en cuenta que nuestra muestra es pequeña, por lo que sería recomendable repetir el estudio con muestras mayores para verificar los resultados.

\section{Conclusiones}

Nuestros pacientes se estaban dializando con presiones dinámicas por encina de los márgenes sugeridos como seguros, por lo que es recomendable controlar el flujo de bomba con el fin de ajustar estas presiones.

Adaptarnos, implica una disminución del flujo de bomba que afecta negativamente a la dosis de diálisis, por lo que sería necesario realizar cambios de pautas de manera individualizada para recuperar la eficacia dialítica perdida.

Recibido: 15 agosto 16

Revisado: 18 agosto 16

Modificado: 20 agosto 16

Aceptado: 27 agosto 16

\section{Bibliografía}

1. Sociedad Española de Nefrología. Guías de Acceso Vascular en Hemodiálisis.2004.

2. López-Menchero Martínez R, Herrero-Calvo JA, Fernández-Rivera $\mathrm{C}$. Problemas actuales en el acceso vascular para hemodiálisis. Angiología. 2005; 57 (2): 219-226.

3. Konner K, Nonnast-Daniel B, Ritz E. The arteriovenous fistula. J Am Soc Nephrol .2003; 14: 1669-80.

4. Rodríguez JA, González Parra E. Accesos vasculares para hemodiálisis: preparación del paciente con insuficiencia renal crónica. Angiología. 2005;57 (2): 11-21.

5. National Kidney Foundation. KDOQI Clinical Practice Guidelines and Clinical Practice Recommendations for 2006 Updates: Haemodialysis Adequacy, Peritoneal Dialysis Adequacy and Vascular Access. Am J Kidney Dis 2006;48(Suppl 1):S1-S322.

6. Miranda-Camarero VM. Cuidados de las fístulas arteriovenosas. Intervenciones y actividades del profesional de enfermería. Dial Traspl. 2010; 31 (1): 12-16.

7. Galera-Fernández A, Martínez-de Merlo MT, Ochando-García A. Accesos vasculares para hemodiálisis: cuidados de enfermería. Angiología 2005; 57 (Supl 2): S159-S168.

8. Cirera Segura F, Reina Neyra E.M- Fernández-Aramburu,T.L.,Ceballos Camas R.Actuaciones de Enfermería sobre los accesos vasculares internos y su influencia en la calidad de diálisis. Disponible en: http://www.revistaseden.org/files/122.pdf.

9. Rodríguez JA, Ferrer E, Olmos A, Codina S, Borrellas $X$, Piera L. Análisis de supervivencia del acceso vascular permanente. Nefrología 21: 260-273; 2001.

10. Márquez Benítez J, Ricci Valero L, Calderón Lozano $F$, Ruiz Jiménez AB. Supervivencia y comorbilidad en los pacientes que inician hemodiálisis crónica. Disponible en: http://www.revistaseden.org/ files/266a.pdf.

11. Sanchez Villar I, Cabello 0, Valido P y cols. Supervivencia de la FAVI y sesión de hemodiálisis ¿Cómo se relacionan?. Libro de comunicaciones del XXXIII Congreso de la Sociedad Española de Enfermería Nefrología; San Sebastián oct, 2008: 195-200. 
12. Parisotto MT, Schoder Vu, Miriunis $C$, Grassmann AH, Scatizzi LP, Kaufmann P, Stopper A, Marcelli D. Cannulation technique influences arteriovenous fistula and graft survival. Kidney International 2014, 86 (4): 790-797.

13. Maduell F, Navarro V.Medida y control de la eficacia en hemodiálisis. Diálisis adecuada. Monitorización contínua. Tratado de Hemodiálisis Editorial Médica JIMS S.L., Barcelona, pgs 243-270, 2006.

14. Lowrie EG, Chertow GN, Lew NM y col. The urea (clearance $\mathrm{x}$ dialysis time) product (Kt) as an outcome- based measure of hemodialysis dose. Kidney Int 1999; 56: 729-737.

15. Lowrie EG, Li Z, Ofsthun NJ, Lazarus JM. Evaluating a new method to judge dialysis treatment using online measurements of ionic clearance. Kidney Int 70: 211-217, 2006.

16. Maduell F, Vera M, Serra N y cols. Kt como control y seguimiento de la dosis en una unidad de hemodiálisis. Nefrología 2008; 28: 43-47.

17. Fernández AV, Soto $S$, Arenas $M$ y cols. Estudio comparativo de la dosis de diálisis medida por dialisancia ionica (Kt) y por Kt/V. 21 Rev Soc Esp Enferm Nefrol 2009;12 (2):97-102.

18. Ortiz-Herrasti E, Martínez Cercós AC, García León $\mathrm{J}$ y cols. Control clínico del acceso vascular. Angiología 2005, 57 (Supl 2): 583-592.

19. Kamur V, Dopan T, Besarab A y cols. Accesos vasculares para hemodiálisis. Dauguirdas JT, Manual de Diálisis $4^{a}$ Edición. Ed Wolters Klisner. 2008. Pg 104-123.

20. Held PJ, Port FK, Wolfe RA, Stannard DC, Carrol CE, Dagirdas JT, Bloembergen WE, Greer JW, Hahim RM: The dose of hemodialysis and patients mortality. Kidney Int 50: 550-556, 1996.

21. Collins $A$, Liao $M$, Umen A: High-efficiency treatmentsusing conventional equipment. En Hemodialysis highefficiency treatments. Churchill Livingstone Inc, pp 91-104, 1993.

22. Maduell F, García M, Alcázar R. Dosificación y adecuación del tratamiento dialítico. Guías SEN: Guías de Centros de hemodiálisis. Nefrologia 2006; 26 (Suppl 8): 15-21.

23. James Tattersall, Alejandro Martin-Malo, Luciano Pedrini, Ali Basci, Bernard Canaud, Denis Fouque,
Patrick Haage, Klaus Konner, Jeroen Kooman, Francesco Pizzarelli, Jan Tordoir, Marianne Vennegoor, Christoph Wanner, Piet ter Wee, and Raymond Vanholder. EBPG guideline on dialysis strategies. Nephrol Dial Transplant 2007; 22 (Suppl 2): ii5ii21.

24. E. Gallego, J. M. Portolés, F. Llamas, A. Serrano, S. Tallón, E. Andrés, C. Gómez, E. Olivas y L. Sánchez Tárraga. Efecto del calibre de las agujas sobre la recirculación y la eficacia de la hemodiálisis. Nefrologia. Vol. XVII. Núm. 4. 1997.

25. Crespo R, Casas R, Muñoz J, Rivero $F$, Contreras MD, Muñoz L, López E. Influencia del calibre de la aguja sobre el grado de dolor originado en la punción de la fístula arteriovenosa. Disponible en: http://www.revistaseden.org/files/Influencia \%20 del $\% 20$ calibre $\% 20$ de $\% 201$ a $\% 20$ aguja $\% 20$ sobre $\% 20$ el\%20grado $\% 20$ de $\% 20$ dolor $\% 20$ originado\%20en\%20la\%20punción\%20de\%20 la\%20fístula\%20arteriovenosa.pdf.

26. Aznar S, Cegarra RB, Badallo Mira, Pagán D, Bartolomé A. Estudio comparativo en pacientes en hemodiafi Itración on-line postdilucional de alta eficacia con diferentes flujos de líquido dializante con tiempo programado y tiempo real. 63 Enferm Nefrol 2013; 16 Suppl (1): 40/121.

27. Rodríguez Gayán $P$, Conde Martínez M, Díaz de Argote Cervera P, Martínez Aranda MA. ¿Usamos el flujo de baño óptimo en hemodiálisis y hemodiafiltración on-line?. Enferm Nefrol [Internet]. 2014 [citado 2016 Abr 22]; 17( Suppl 1 ): 44-44. Disponible en: http://scielo.isciii.es/scielo.php?script=sci_arttext\&pid=S2254-28842014000500030\&In$g=e s$.

28. Fernández $A V$,Pérez $L$,Fernández Caro J, Ruiz M, Horrillo F, Caparros V, García F, Vilar MV. Influencia en la dosis de diálisis de diferentes flujos de líquido dializante en el paciente tratado con hemodiafiltración on-line o hemodiálisis convencional. Rev Soc Esp Enferm Nefrol 2011; 14 (1):37/42.

29. Maduell F, Arias M. Dosis de diálisis. doi:10.3265/ Nefrologia.2010.publ.ed80.chapter2811. Disponible en: http://www.revistanefrologia.com/esmonografias-nefrologia-dia-articulo-dosis-dialisis-36\#biblio. 